\title{
miR-124 Suppresses Pancreatic Ductal Adenocarcinoma Growth by Regulating Monocarboxylate Transporter 1-Mediated Cancer Lactate Metabolism
}

\author{
De-Hai Wu Hao Liang $^{\mathrm{b}}$ Shou-Nan Lu ${ }^{\mathrm{a}}$ Hao Wang ${ }^{\mathrm{a}}$ Zhi-Lei Su ${ }^{\mathrm{a}}$ Lei Zhang ${ }^{\mathrm{c}}$ \\ Jian-Qun Mab Mian Guo ${ }^{d}$ Sheng Tai ${ }^{a}$ Shan Yu \\ aDepartment of General Surgery, the Second Affiliated Hospital of Harbin Medical University, Harbin, \\ bDepartment of Esophageal Mediastinum, Harbin Medical University Cancer Hospital, Harbin, \\ 'Department of Pathology, Harbin Medical University, Harbin, 'Department of Neurosurgery, the \\ Second Affiliated Hospital of Harbin Medical University, Harbin, eDepartment of Pathology, the Second \\ Affiliated Hospital of Harbin Medical University, Harbin, China
}

\section{Key Words}

microRNA-124 - Monocarboxylate transporter 1 - Lactate metabolism - Pancreatic ductal adenocarcinoma

\begin{abstract}
:
Background/Aims: Increasing evidence shows that reprogramming of energy metabolism is a hallmark of cancer. Considering the emergence of microRNAs as crucial modulators of cancer, this study aimed to better understand the molecular mechanisms of miR-124 in regulating glycolysis in human pancreatic cancer. Methods: RT-PCR was used to investigate the expression of monocarboxylate transporters (MCTs) in pancreatic ductal adenocarcinoma (PDAC) patient samples and the PANC-1 cell line. A public database and immunochemistry were used for comprehensive analysis of MCT1 expression. The targeting of MCT1 by miR124 was predicted by software and validated for the MCT1 3'-UTR by dual-luciferase reporter analysis. Cell proliferation, apoptosis, migration, xenografting, and the intracellular $\mathrm{pH}$ and $L$-lactate levels were assessed. Hypoxia-inducible factor- $\alpha(\mathrm{HIF}-1 \alpha)$ and lactate dehydrogenase A (LDH-A) expression levels were determined by RT-PCR and western blotting. Results: MCT1 expression was higher in PDAC tissue than in normal tissue. Inhibition of MCT1 affected lactate metabolism, resulting in a higher intracellular $\mathrm{pH}$ and less proliferation of PANC-1 cells. MCT1 was the target gene of miR-124. In in vitro experiments, miR-124 inhibited the glycolytic activity of PANC-1 cells by targeting MCT1, further decreasing the tumor phenotype by increasing the intracellular $\mathrm{pH}$ through LDH-A and HIF-1 $\alpha$. In in vivo experiments, overexpression of miR-124 and silencing of MCT1 significantly inhibited tumor growth. Conclusion: miR-124 inhibits

D.-Hai. Wu and $\mathrm{H}$. Liang contributed equally to this work.

\begin{tabular}{ll}
\hline Sheng Tai & Department of General Surgery, Department of Pathology, the Second Affiliated Hospital \\
and Shan Yu & of Harbin Medical University, Harbin 150086 (China) \\
& Tel. +86-451-86605356, Fax+86-451-86605356, E-Mail taisheng1973@163.com; yushan@hrbmu.edu.cn
\end{tabular}
\end{abstract}


the progression of PANC-1 by targeting MCT1 in the lactate metabolic pathway. Our findings provide novel evidence for further functional studies of miR-124, which might be useful for future therapeutic approaches to PDAC.

\section{Introduction}

Pancreatic cancer lacks clinical symptoms and is often diagnosed at an advanced stage. The mortality rate of pancreatic cancer, a highly malignant disease of the digestive system, has been increasing in recent years [1]. Pancreatic ductal adenocarcinoma (PDAC) is the most common type of pancreatic cancer, accounting for over $80 \%$ of cases. PDAC originates in the epithelial cells of the pancreatic duct, and its consistently aggressive behavior results in a 5-year survival rate of 5\% [2]. Although targeted therapy has proven effective for other digestive tract cancers, the therapeutic results were quite limited in late-stage clinical trials for PDAC [3]. Surgical resection is considered the only chance of a cure for pancreatic cancer; however, fewer than $20 \%$ of patients have the opportunity to undergo surgery because of their late clinical diagnosis [4]. Other chemotherapeutic options, including gemcitabine, which is believed to improve the survival rate for PDAC, are limited by drug resistance and side effects $[5,6]$.

MicroRNAs (miRNAs) are evolutionarily conserved, single-stranded noncoding short RNAs consisting of 18-24 nucleotides. They participate in post-transcriptional regulation by targeting the $3^{\prime}$ untranslated regions (UTRs) of target genes. miRNAs are believed to play a crucial role in the pathogenesis and progression of PDAC and are considered promising targets for RNA interference (RNAi)-based therapeutics.

Reprogramming of energy metabolism is a hallmark of cancer $[7,8]$ and lactate is believed to play a crucial role in tumor glycolysis metabolism [9]. Early evidence showed that monocarboxylate transporter 1 (MCT1)-mediated small molecule transportation is an effective strategy to control glycolytic tumor metabolism [10]. PDAC cells are a type of malignant tumor cell and heavily rely on glycolysis for their growth. miRNAs are emerging as key regulators that target metabolic enzymes, oncogenes, and tumor suppressors in metabolic pathways [11]. miR-124, which targets Rac1, can be downregulated by methylation, resulting in pancreatic cancer progression and metastasis [12]. The negative regulation by miR-124 of MCT1 was identified in malignant medulloblastomas [13]. However, our understanding of the role of miR-124 in PDAC remains very limited, particularly its role in cell glycolysis metabolism.

In this study, we found that miR-124 inhibited the glycolytic activity of PANC- 1 cells by targeting MCT1, further decreasing the tumor phenotype in in vitro and in vivo experiments. miR-124 was also able to alter the acidity of PANC-1 cells and inhibit the activity of hypoxiainducible factor- $\alpha$ (HIF- $\alpha$ ) through the regulation of MCT1, but this regulation did not lead to apoptosis. Therefore, the results of this study show that miR-124 regulates the metabolic pathway by targeting MCT1 and suggests a potential therapeutic target for pancreatic cancer patients.

\section{Materials and Methods}

Patient samples and cell lines

Five PDAC samples with a definite pathological diagnosis were collected from the Second Affiliated Hospital of Harbin Medical University. With the approval of the Ethics Committee of Harbin Medical University, all patients were informed of the purpose of sample collection and provided written informed consent. PANC-1 and 293T cells were obtained from the American Type Culture Collection (ATCC, Manassas, VA) and cultured in DMEM (HyClone, Logan, UT) supplemented with 10\% fetal bovine serum (Gibco, Gaithersburg, $\mathrm{MD}$ ) at $37^{\circ} \mathrm{C}$ in a $5 \% \mathrm{CO}_{2}$ incubator.

\section{KARGER}




\section{Cellular Physiology Cell Physiol Biochem 2018;50:924-935 \begin{tabular}{ll|l} 
DOl: 10.1159/000494477 & $\begin{array}{l}\text { O 2018 The Author(s). Published by S. Karger AG, Basel } \\
\text { www.karger.com/cpb }\end{array}$
\end{tabular}}

Wu et al.: Mir-124 Suppressed Cancer by MCT1

\section{Bioinformatic analyses}

The PDAC database was extracted from OncoLnc (http://www.oncolnc.org/), and an MCT expression heat map and bar chart were made for 52 patients. miRNA target prediction analysis was performed using three online prediction programs: TargetScan (www.targetscan.org), MiRanda (www.miranda.org), and miRBase (www.mirbase.org). StarBase was used to demonstrate the association between miR-124-3p and SCL16A1 (MCT1) from the median value of 151 pancreatic cancer samples (http://starbase.sysu.edu.cn/). The immunohistochemical patterns of MCT1 in pancreatic cancer and normal tissues are from the human protein atlas (www.proteinatlas.org).

\section{Real-time PCR}

Total RNA of patient samples and the PANC-1 cell line was extracted by using TRIzol reagent (Invitrogen, Carlsbad, CA) according to the manufacturer's protocol. For miRNA reverse transcription, $1 \mu \mathrm{g}$ of total RNA was reverse-transcribed into cDNA by using the All-in-One miRNA First-Strand cDNA Synthesis Kit (GeneCopoeia, Rockville, MD). For mRNA reverse transcription, a Roche first-strand synthesis kit was used (Roche, Philadelphia, PA). RT-PCR was performed in triplicate using the SYBR Green Master Mix Kit (Roche). Beta-actin and U6 were used as internal controls for mRNA and miRNA RT-PCR, respectively. The $2^{-\Delta \Delta c t}$ method was used to analyze the relative levels of gene expression.

The following primers were used:

MCT1, forward 5'-GTGGCTCAGCTCCGTATTGT-3',

reverse 5'-GAGCCGACCTAAAAGTGGTG-3';

MCT2, forward 5'-CAACACCATTCCAAGACAGC-3',

reverse 5'-TGGCTGTTATGTACGCAGGA-3';

MCT3, forward 5'-GGATGTGTTGAAGAACTATGAGATC-3',

reverse 5'-CCGGGTTCCTCTGCAACA-3';

MCT4, forward 5'-CAGTTCGAGGTGCTCATGG-3',

reverse 5'-ATGTAGACGTGGGTCGCAT-3',

LDH-A, forward 5'-TCTCTGTAGCAGATTTGGCAGA-3',

reverse 5'-AAGACATCATCCTTTATTCCGTAAA-3'

HIF- $1 \alpha$, forward 5'-TTTTCAAGCAGTAGGAATTGGAA-3',

reverse 5'-GTGATGTAGTAGCTGCATGATCG-3'.

miR-124-3p: sense, 5'-GCTAAGGCACGCGGTG-3';

antisense, 5'-GTGCAGGGTCCGAGGT-3'.

The reference gene primers used were as follows: U6, sense 5'-CTCGCTTCGGCAGCACATATACT-3', antisense, 5'-ACGCTTCACGAATTTGCGTGTC-3'; and $\beta$-actin, sense 5'-AGGGGCCGGACTCGTCATACT-3', antisense $5^{\prime}$-GGCGGCACCACCATGTACCCT-3'.

\section{Immunohistochemistry}

The PDAC patient samples were fixed in $10 \%$ formalin for $12 \mathrm{~h}$, embedded in paraffin, and cut into $4-\mu \mathrm{m}$ sections. After deparaffinization in xylene, the sections were subjected to antigen retrieval involving 20 min of microwave boiling, incubated with $3 \%$ hydrogen peroxide for $10 \mathrm{~min}$, and blocked with $10 \%$ goat serum for $1 \mathrm{~h}$. The sections were then incubated with 1:200 diluted MCT1 antibody (\#85021, Abcam, Cambridge, MA) overnight at $4^{\circ} \mathrm{C}$. Secondary antibody (biotinylated anti-rabbit IgG; 1:200 dilution; Maxim Biotechnology, Fuzhou, China) was added and incubated for $30 \mathrm{~min}$ at room temperature. Then, the sections were incubated with 3\% diaminobenzidine as a chromogenic substrate for $5 \mathrm{~min}$.

\section{Transient transfection}

PANC-1 cells with $80 \%-90 \%$ confluence were transfected with miR-124 mimic or si-MCT1 or nontarget control (NC) using Lipofectamine 2000 (Invitrogen) in accordance with the manufacturer's protocol. The miR-124-3p mimic sequence was 5'-UAAGGCACGCGGUGAAUGCC and the miR-29c mimic sequence was 5'-UAGCACCAUUUGAAAUCGGUUA. hMCT1 small interfering (si)RNA (Qiagen, Hilden, Germany) was synthesized with the following sequence: AAGAGGCUGACUUUUCCAAAU. The AllStars Negative Control siRNA (Qiagen) was used as a non-target control. The synthesized sequence was UUCUCCGAACGUGUCACGU. 


\section{Cellular Physiology Cell Physiol Biochem 2018;50:924-935 \begin{tabular}{ll|l} 
DOl: 10.1159/000494477 & $\begin{array}{l}\text { O 2018 The Author(s). Published by S. Karger AG, Basel } \\
\text { www.karger.com/cpb }\end{array}$
\end{tabular}}

Wu et al.: Mir-124 Suppressed Cancer by MCT1

Western blotting

The total protein concentration was determined by the bicinchoninic acid method [14]. Total protein was separated by $10 \%$ sodium dodecyl sulfate-polyacrylamide gel electrophoresis and transferred to polyvinylidene fluoride membranes (Pall Life Sciences, Port Washington, NY). The membranes were blocked with $5 \%$ fat-free milk and TBST (Tris-buffered saline with $0.1 \%$ Tween-20) for $2 \mathrm{~h}$ at room temperature; subsequently, primary antibodies (Cell Signaling Technology, Danvers, MA) and TBST were incubated with the membrane at $4^{\circ} \mathrm{C}$ overnight. After the secondary antibody was applied, the bands were detected by chemiluminescence.

\section{Determination of the intracellular $\mathrm{pH}$ and L-lactate levels}

After silencing of MCT1, we used 2'7'-bis-(2-carboxyethyl)-5-(and-6)-carboxyfluorescein, acetoxymethyl ester (BCECF-AM, Invitrogen) to evaluate the $\mathrm{pH}$ level. The lactate concentration was determined by the colorimetric method according to the manufacturer's protocol [15]. L-lactic acid (10 mM; Sigma, St. Louis, MO) was added to PANC-1 cells, which were washed twice with phosphate-buffered saline supplemented with $10 \mathrm{mM}$ D-lactic acid and then lysed in $200 \mu \mathrm{l}$ of ice-cold RIPA buffer. The sample was filtered through a spin column with a 10-kDa cut-off. The L-lactate concentrations of the supernatant lysates were quantified using a specific enzyme assay and an ISCUS flex analyzer.

\section{Dual-luciferase reporter gene assay}

A total of 500 ng of wild-type or mutant pMIR-EXPORT-MCT1-3'-UTR plasmid and pRL-SV40 vector were transiently cotransfected into HEK293T cells with 60 pmol of miR-124-3p mimics or nontargeted control in a 24-well plate. Firefly luciferase was used as a reporter gene to measure the expression of MCT1 under the experimental conditions, and a control reporter gene was used as an internal control to normalize the results of the experimental reporter test. Cells were harvested $24 \mathrm{~h}$ post-transfection, and the firefly and Renilla luciferase activities were evaluated by a dual-luciferase reporter (Promega, Madison, WI).

\section{MTT assay}

PANC-1 cells were seeded at $3 \times 10^{3}$ cells per well in a 96-well plate. After transfection with siRNA for MCT1 or miRNA mimics for $24 \mathrm{~h}, 48 \mathrm{~h}$, and $72 \mathrm{~h}$, a Vybrant MTT Cell Proliferation Assay Kit (Thermo Fisher Scientific, Waltham, MA) was used according to the manufacturer's protocol, and the optical density was measured.

\section{Wound-healing assay}

PANC-1 cells were transfected with si-MCT1 or miR-124-3p mimic for $24 \mathrm{~h}$ and then subjected to a previously described protocol for assessing wound healing [16].

\section{Transwell assay}

Cell migration was determined after a 1-day transfection by using Transwell insert chambers (Corning, Corning, NY) as described previously [17]. After being seeded in the upper chamber for $24 \mathrm{~h}$, cells that had migrated through the membrane were fixed with methanol, stained with $0.1 \%$ crystal violet (Beyotime, Shanghai, China), imaged, and counted.

\section{Apoptosis assay}

After MCT1 overexpression and interference with miR-124, flow cytometry was used to evaluate the apoptosis of PANC-1 cells with Annexin V-FITC/PI (Thermo Fisher Scientific) according to the manufacturer's protocol [18].

\section{Xenograft experiments}

We used the method described in the literature to construct a lentiviral pLu-Puro-Indu-shRNAmiR-124/MCT1 vector [19]. PANC-1-derived stable cell lines were selected and confirmed by RT-PCR. Nine NPG mice were randomly divided into three groups. Subsequently, stable cells were collected and mixed with 5\% Matrigel, and each mouse was subcutaneously injected in the hind leg with $2 \times 10^{6}$ cells. The mice were sacrificed on day 28 after injection, and the tumors were removed and imaged. We calculated tumor volumes using the formula $\mathrm{V}=0.52 \times$ diameter $\left(\mathrm{mm}^{3}\right)[20]$. 
Statistical methods

SPSS 18.0 statistical software (SPSS Inc., Chicago, IL) was used to perform statistical analysis of the data. All experimental data are expressed as the mean \pm standard deviation, and the value of each group was analyzed with the assumption of homogeneity of variance. Comparisons between the two groups were performed using the Student's t-test. $P<0.05$ was considered statistically significant.

\section{Results}

\section{MCT1 is highly expressed in PDAC}

The GEO database showed that MCT1, MCT3, and MCT4, which are among the four genes in the SLC16A family, are more highly expressed in pancreatic cancer than in normal tissue (Fig. 1A). Metabolic Gene Rapid Visualizer software demonstrated that SLC16A1 (MCT1) exhibited a higher expression level in tumor tissues than in normal tissues, but MCT2 showed a lower expression level (Fig. 1B). The relative mRNA expression levels of MCT1 were significantly higher in PDAC samples than those of MCT2, MCT3, and MCT4, as

Fig. 1. MCT1 was upregulated in human PDAC. (A) Expression level changes of MCT family genes involved in the energy metabolism for PC and normal tissues. Each column represents expression changes of a gene. The fold change of gene expression is color-coded with pink, white and blue for up-, no and down-regulation. (B) The differential expression levels of four SLC16 family genes in cancer and normal tissues were identified by Metabolic Gene Rapid Visualizer software. (C) Five PDAC tissues and PANC-1 cells were used to identify the relative expression level of MCT family genes by RT-qPCR $(\mathrm{n}=5 ; \mathrm{P}<0.001)$. (D) From the website (www. oncolnc.org), among 52 patients with pancreatic cancer, 26 patients revealed a high expression level of MCT1 and exhibited a significantly lower survival rate compared with patients with low expression levels. (E) Immunohistochemical analysis showed the expression of MCT1 in cancer and para-cancerous. The left and right sides were $20 \times$ and $40 \times$ microscopic results. The direction of the triangular arrow indicated the expression level of MCT1. (F) The data showed the expression of MCT1 in PCs and normal tissues by using immunohistochemical results (www.proteinatlas.org).

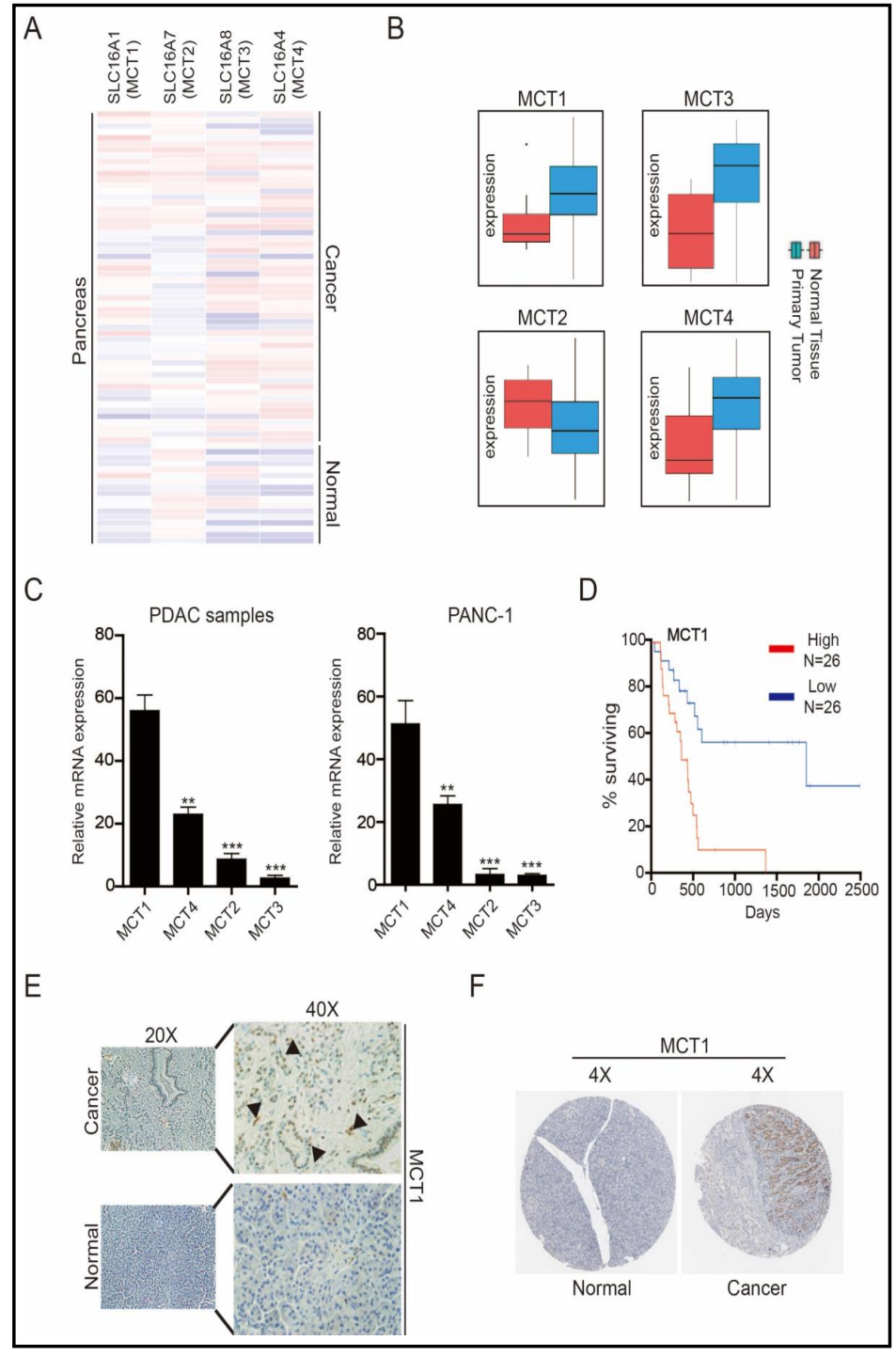




\section{Cellular Physiology Cell Physiol Biochem 2018;50:924-935 \begin{tabular}{ll|l} 
DOl: 10.1159/000494477 & $\begin{array}{l}\text { O } 2018 \text { The Author(s). Published by S. Karger AG, Basel } \\
\text { www.karger.com/cpb }\end{array}$
\end{tabular} \\ Wu et al.: Mir-124 Suppressed Cancer by MCT1}

determined by RT-qPCR. Similar results were found in PANC-1 cells (Fig. 1C). Data from 52 PDAC patients were obtained from the OncoLnc website (www.oncolnc.org). The survival rate was lower for patients with higher expression of MCT1 $(n=26)$ than for those with lower expression levels $(n=26)$ (Fig. 1D). MCT1 protein was detected in the cell membrane and nucleus in five PDAC tissue samples by immunohistochemistry (Fig. 1E). The website data showed that MCT1 was highly expressed in pancreatic cancer, which was consistent with our immunohistochemical results (Fig. 1F).

\section{Inhibition of MCT1 affects lactate metabolism}

The downregulation of MCT1 expression by si-MCT1 was confirmed by RT-qPCR and western blot analysis (Fig. 2A). MTT results showed that higher cell si-MCT1 concentrations resulted in fewer cells, indicating the role of MCT1 in cell proliferation (Fig. 2B). The intracellular L-lactate concentration of PANC-1 cells was significantly increased 24 and $48 \mathrm{~h}$ after transfection with si-MCT1 (Fig. 2C). Measurements of the pH showed that the fluorescence intensity of the si-MCT1 group was lower than that of the si-NC group after $12 \mathrm{~h}$ (Fig. 2D). Transfection with si-MCT1 inhibited the LDH-A expression in PANC-1 cells.

Fig. 2. Inhibition of MCT1 affects lactate metabolism. (A) The efficiency of siMCT1 downregulating was verified by RT-qPCR and Western Blot. (B) The absorbance value of PANC1 cells after transfected by si-MCT1 or NC was demonstrated by MTT method. (C) The level of intracellular L-lactate was determined in PANC-1 cells transfected with si-MCT1, the alterations in lactate values were observed at 24 and $48 \mathrm{~h}$. (D) The relation between the fluorescence intensity and $\mathrm{PH}$ value of PANC-1 cells were identified using by BCECFAM. The fluorescence of PANC-1 cells was observed in the si-MCT1 group and NC group at $0 / 24$ h. (E) Lactate and pyruvate conversion requires the participation of LDH-A and $\mathrm{LDH}-\mathrm{B}$, the relative expression level of LDH-A was determined by RTqPCR. (F) PCR was used to verify that MCT1 stimulated HIF- $\alpha$ by targeting lactate. PANC-1 cells were divided

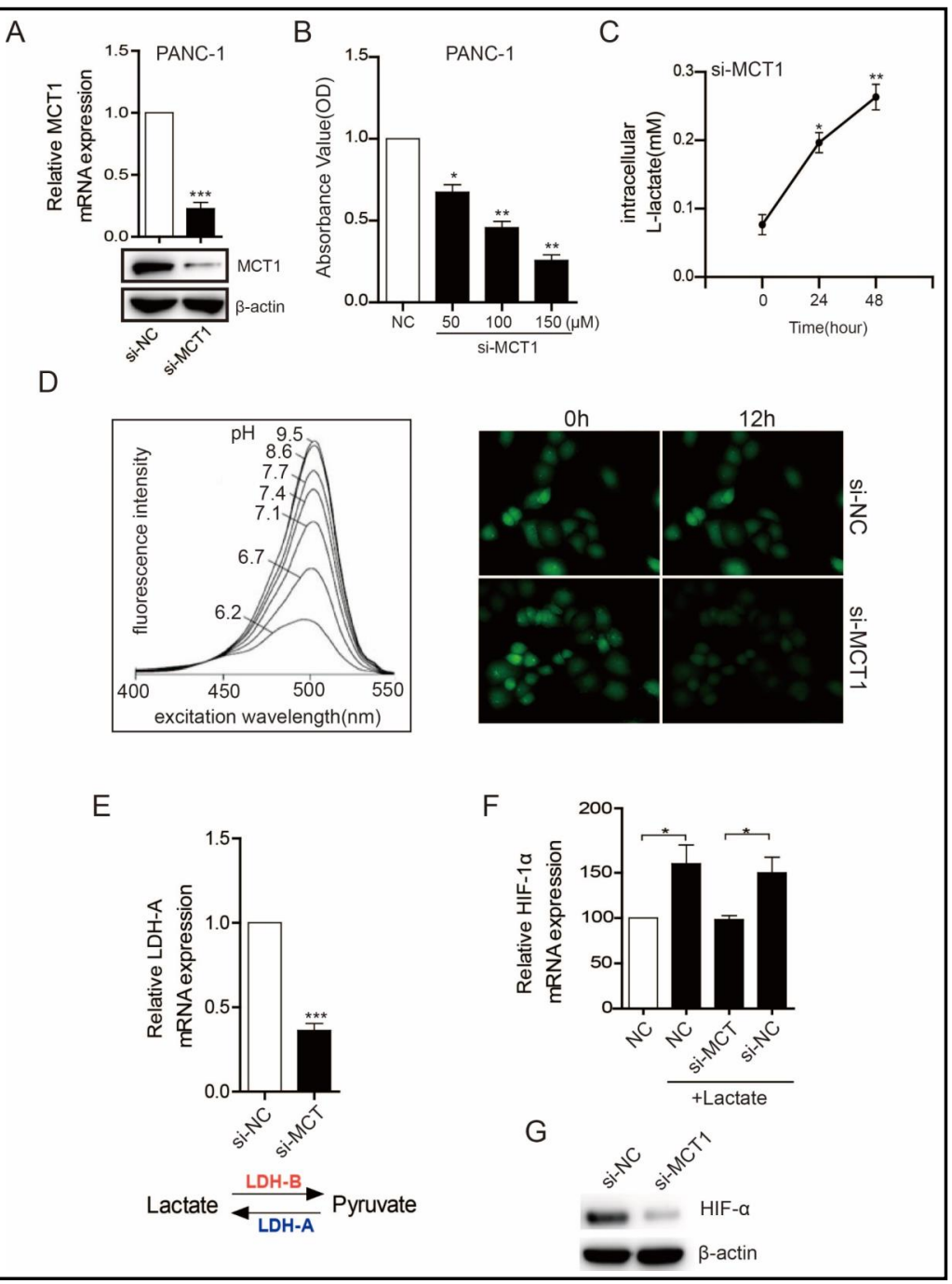
into four groups: NC (negative group), NC (lactate was added), si-MCT1 (transfected si-RNA matched MCT1, lactate), si-NC (transfected si-RNA unrelated MCT1). (G) Western blot analysis was performed to identify MCT1 and the expression level of HIF- $\alpha$. $\beta$-actin was used as the control. 
Lactate and pyruvate conversion measurements tended to indicate more lactate (Fig. 2E). In addition, the RT-PCR results demonstrated that MCT1 upregulated HIF- $\alpha$ expression via lactate, and western blot analysis showed that intracellular HIF- $\alpha$ expression was inhibited by si-MCT1 (Fig. 2F, G).

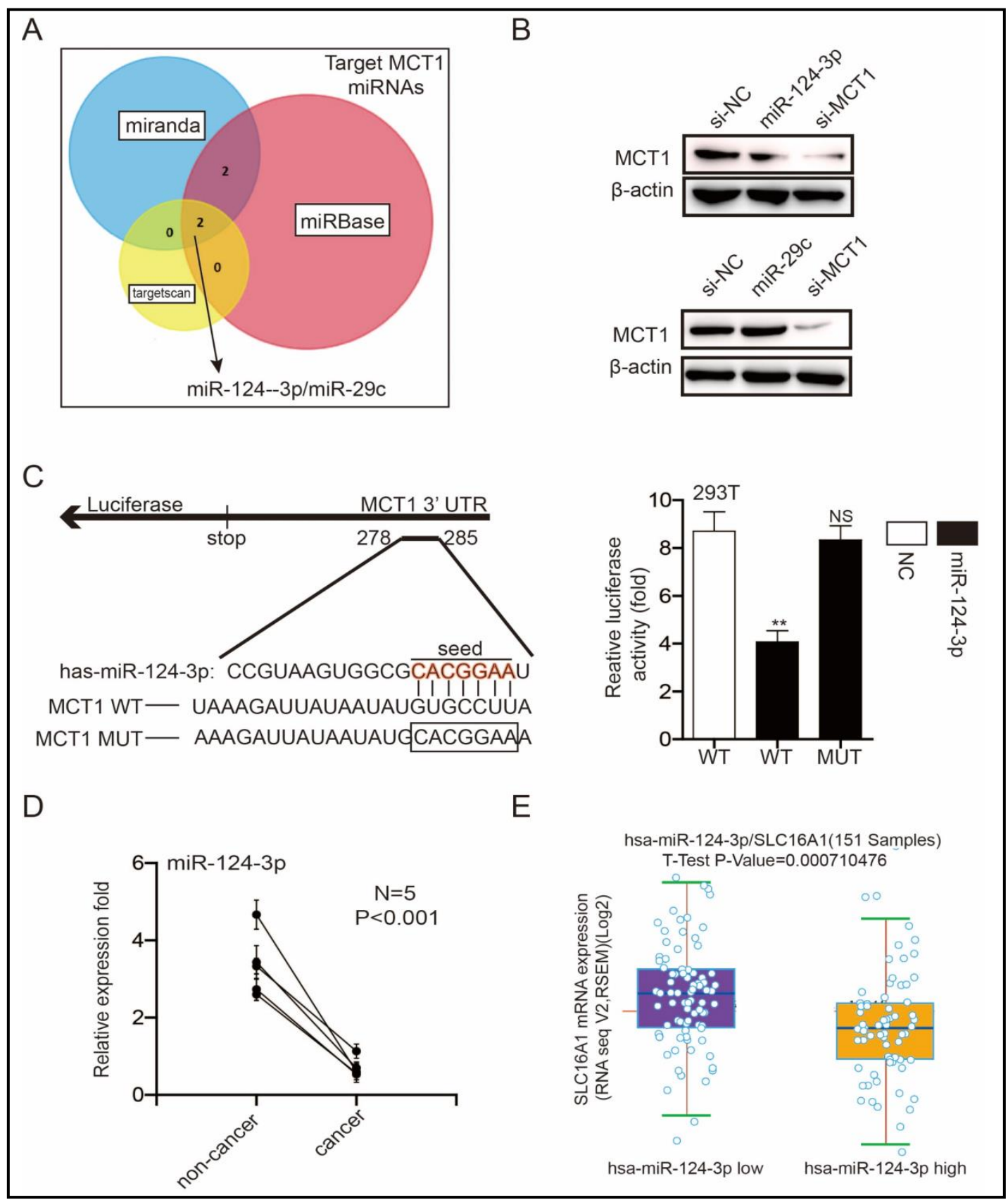

Fig. 3. Bioinformatics prediction and miRNA-124 validation. (A) Three software were used to predict the miRNA targeting MCT1. The prediction identified miR-124 and miR-29c. (B) Western blot was used to evaluate the expression of MCT1 in miR-124 and miR-29c transfected cells. (C) Diagram of the MCT1-3'-UTR with potential binding-sites for miR-124 and the seed binding sequences of miR-124 with MCT1. Relative luciferase activity of reporters including wildtype (WT) or mutant (MUT) MCT1-3'UTR co-transfected with NC or miR-124 mimics. ${ }^{* *} \mathrm{P}<0.01$. (D) RT-qPCR was used to detect the expression of miR-124 in cancer and adjacent tissues $(\mathrm{P}<0.001)$. (E) StarBase analysis showed the relationship between MCT1 and miR-124 expression by using T-test in $151 \mathrm{PC}$ samples. $\mathrm{P}=0.0007$. 


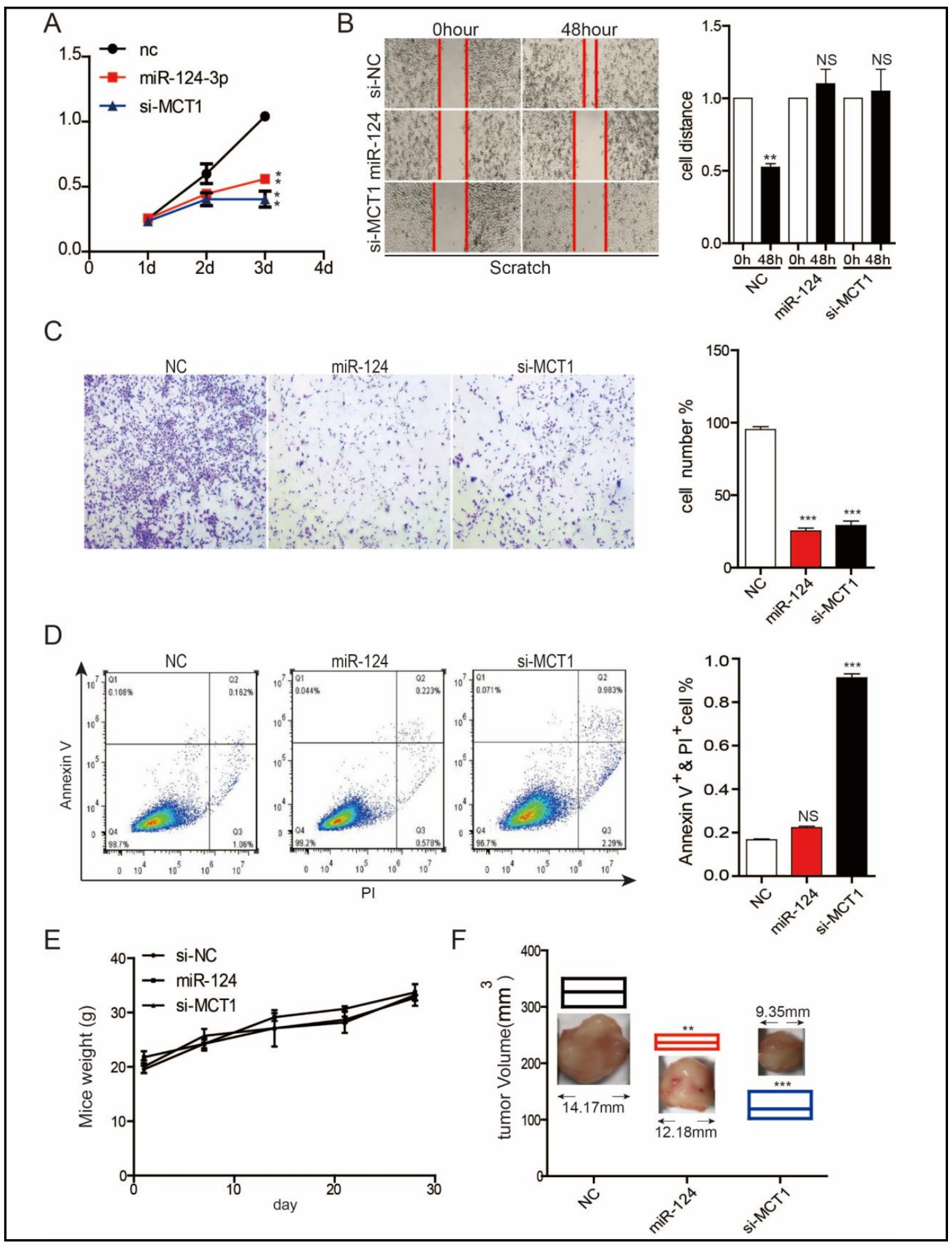

Fig. 4. miR-124 inhibits progression of PDAC via MCT1 in vitro and vivo. (A) si-MCT1, miR-124 mimics or NC plasmid were transfected into PANC-1 cells and the cell proliferation rate was determined by MTT assay. (B) Wound healing assay of PANC-1 cells transfected with si-MCT1, miR-124 mimics or si-NC plasmid. Representative images are presented at 0 and $48 \mathrm{~h}$ following the scratch. (C) Transwell assay of PANC-1 cells transfected with miR-124 mimics, si-MCT1 or NC. Cell numbers are presented in a graph. ${ }^{* * *} \mathrm{P}<0.001$. (D) The effect of miR-124 overexpression on apoptosis of PANC-1 cells was followed by staining with 10 $\mu \mathrm{l}$ of Annexin V-FITC and $10 \mu \mathrm{l}$ of PI for 24 hours. The effect of miR-124 on the apoptosis rate was further determined by flow cytometry. (E) In vivo, changes in mice weight were monitored during tumor formation. (F) In vivo, the tumor volume of mice was measured after 28 days. ${ }^{* *} \mathrm{P}<0.05$, ${ }^{* * *} \mathrm{P}<0.001$.

\section{KARGER}




\section{MCT1 is the target gene of miR-124}

miR-124 and miR-29c were identified to target MCT1 by using three different analytical programs (Fig. 3A). Western blot analysis revealed that overexpression of miR-124 inhibited MCT1 expression, whereas miR-29c overexpression did not (Fig. 3B). HEK293T cells were transfected with the mutant (MUT) and wild-type (WT) 3'-UTR of MCT1. The luciferase activity of cells transfected with the WT was inhibited by miR-124; however, the activity was restored by MUT transfection (Fig. 3C). RT-qPCR analysis demonstrated that miR-124$3 p$ expression was upregulated in five PDAC patient tumor samples compared with paired noncancerous tissue (Fig. 3D). Data from starBase showed that the miR-124 level was inversely proportional to the MCT1 level (Fig. 3E).

\section{miR-124 inhibits the growth of PDAC via MCT1 in vitro and in vivo}

In vitro, the growth rates of cells were significantly lower in the miR-124 mimic and siMCT1 groups than in the NC group after $72 \mathrm{~h}(\mathrm{P}<0.05$; Fig. 4A). Analysis using an inverted microscope and Image J software revealed that the migration abilities of the si-MCT1 and miR-124 groups were significantly lower than that of the si-NC group after a 48-h incubation (P < 0.05; Fig. 4B). miR-124 inhibited the invasion of PANC-1 cells (Fig. 4C). Compared with the miR-124 and NC groups, the si-MCT1 group had slightly higher rates of early and late apoptosis; however, neither of these groups had clear apoptosis (Fig. 4D).

In vivo, animal weight grew steadily for several weeks in the three groups of mice, which excluded the possibility that other factors interfered with tumor formation (Fig. 4E). The tumor volumes of the mice were $1.64 \pm 0.09 \mathrm{~cm}^{3}(\mathrm{NC}), 0.87 \pm 0.07 \mathrm{~cm}^{3}(\mathrm{miR}-124)$, and 0.49 $\pm 0.06 \mathrm{~cm}^{3}$ (si-MCT1; Fig. 4F). This analysis revealed that miR-124 and MCT1 knockdown significantly inhibited the growth of PDAC cells in vivo.

\section{Discussion}

Recent studies demonstrated that miRNAs play important roles in cancer through various mechanisms, and miRNAs are thus attracting increasing attention. The roles of many miRNAs, including miR-124, as tumor suppressors or proto-oncogenes have been redefined and elucidated [21]. For example, miR-124 is downregulated in pleomorphic glioblastoma and degenerative astrocytoma [22]; gene silencing induced by hypermethylation of miR-124 may be involved in the development of gastric cancer [23]; and the expression level of miR124 in breast cancer is downregulated, a change that is associated with the tumor tissue grade [24]. This evidence suggests that silencing of miR-124, a well-conserved miRNA, is closely related to tumorigenesis and development.

Thus, miR-124 may be a potential anti-tumor target and the reactivation of miR-124 expression could be useful for tumor therapy. It has previously been demonstrated that miR-124 has a low expression level in PDAC due to methylation inhibition of its promoter region; miR-124 silencing promotes tumor development by upregulating the expression of Rac1 [12]. The effect of miR-124 on the signaling pathways in tumors has been reported in previous experiments [12, 25-28]. Our experiments uncovered the role of miR-124 in the level of lactate metabolism via targeting of MCT1, broadening the functional reach of this miRNA.

As a consensus has been reached regarding the abnormity of tumor glucose metabolism [29], our research provides a new way to explore the new function of miRNAs as tumor suppressor genes. The public PDAC database showed that MCT1, MCT3, and MCT4 are highly expressed in pancreatic cancer; however, the level of MCT1 was significantly higher than those of the other MCT subtypes in PANC-1 cells. MCT1 is also directly involved in lactate absorption and efflux and is upregulated and used as an independent prognostic marker in a variety of cancers $[30,31]$. Therefore, for this study, we selected MCT1 as the target gene according to the experimental results for the PANC-1 cell line. The other MCTs may also play 


\section{Cellular Physiology Cell Physiol Biochem 2018;50:924-935 \begin{tabular}{ll|l} 
DOI: 10.1159/000494477 & $\begin{array}{l}\text { O } 2018 \text { The Author(s). Published by S. Karger AG, Basel } \\
\text { www.karger.com/cpb }\end{array}$
\end{tabular} \\ Wu et al.: Mir-124 Suppressed Cancer by MCT1}

an important role in cancers [32-34]; however, we did not perform further research on this topic in this study.

Because MCT1 is the most important carrier in lactate transport, we first demonstrated that MCT1 interference significantly decreases the PANC-1 cell phenotype in a concentrationdependent manner and that thisinhibition directly results in intracellular lactate accumulation and a decrease in the $\mathrm{pH}$. Therefore, to verify this hypothesis, we used the BCECF-AM fluorescent probe to detect the relationship between the intracellular fluorescence intensity and acidity under different treatment conditions. We found that MCT1 interference caused a significant reduction in the intracellular $\mathrm{pH}$ value; this acidic environment disrupted the homeostatic stability of the cell environment and gradually led to cell death with toxicity. siMCT1 decreased the mRNA expression level of LDH-A, supporting the role of MCT1 in lactate metabolism to regulate the acidic environment. We believe that the change in acidity is the main reason for the cytostasis and the clear phenotype. However, in this study, there was no further discussion of the downstream regulatory mechanism of cell proliferation inhibition caused by the $\mathrm{pH}$ decline because a follow-up study is underway.

HIF-1 $\alpha$, an important transcription factor that senses intracellular oxygen changes and regulates the intracellular environment, participates in the regulation of multiple cell signaling pathways [35]. We hypothesize that changes in intracellular $\mathrm{pH}$ can affect oxygen metabolism and thus the level of HIF- $1 \alpha$. Accordingly, we have demonstrated that MCT1 interference reduced the expression level of HIF-1 $\alpha$ by using RT-PCR and western blot analysis, and we found that this change can be restored by the addition of lactate. This evidence further confirmed that lactate is not only the product of cell glycolysis, but also an important factor in regulating the intracellular microenvironment of cancer cells. In addition, we demonstrated that miR-124 is an endogenous miRNA that regulates MCT1. However, we ruled out the possibility that miR-29c, a tumor suppressor [36], is the miRNA targeting MCT1 by using western blot analysis.

Our study also found that, compared with overexpression of miR-124, direct inhibition of MCT1 better inhibits cancer cell proliferation and migration and decreases the efficiency of tumor formation; this result was also consistent with the xenograft experimental results. Although si-MCT1 significantly increased the number of apoptotic cells, neither si-MCT1 nor miR-124 overexpression caused significant apoptosis in cancer cells and the cells may have some sort of rescue mechanism that resists changes in this intracellular environment to maintain their own survival. Because miRNAs have many target genes, miR-124 may inhibit cancer proliferation in a variety of ways, and our research results demonstrated just one of the metabolism-related target mechanisms. Our future work will contribute more information to this area.

\section{Conclusion}

This study provides supporting evidence from the viewpoint of cancer metabolism that the tumor suppressor gene miR-124 can inhibit the efflux of lactic acid by targeting the lactate transporter MCT1, causing cell acidification, which can lead to inhibited cell proliferation and migration. Therefore, deregulation of miR-124, as well as other MCT-targeting miRNA candidates, may contribute to the high MCT expression in PDAC patients, and the miR-124/ MCT1 lactate metabolism axis may have potential therapeutic implications in pancreatic cancer.

\section{Abbreviations}

PDAC (Pancreatic ductal adenocarcinoma); MCT1 (monocarboxylate transporter 1); CCK8 (Cell counting kit-8); HIF- $\alpha$ (hypoxia-inducible factor- $\alpha$ ); ATCC (American Type Culture Collection); LDH-A (Lactate dehydrogenase A). 


\section{Cellular Physiology Cell Physiol Biochem 2018;50:924-935

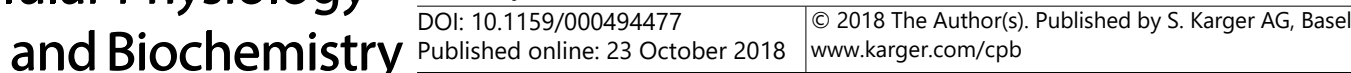

Wu et al.: Mir-124 Suppressed Cancer by MCT1

\section{Acknowledgements}

This project was supported by the National Natural Science Foundation of China (No. 81572472, and 81773161).

The study was approved by the ethics committee of Harbin Medical University. All animal procedures were performed according to national guidelines and approved by the Institutional Committee of Harbin Medical University for Animal Research.

\section{Disclosure Statement}

The authors declare that they have no competing interests.

\section{References}

1 Siegel RL, Miller KD, Jemal A: Cancer Statistics, 2017 CA Cancer J Clin 2017;67:7-30.

2 McGuire S: World Cancer Report 2014 Geneva, Switzerland: World Health Organization, International Agency for Research on Cancer, WHO Press, 2015 Adv Nutr 2016;7:418-419.

-3 Di Marco M, Grassi E, Durante S, Vecchiarelli S, Palloni A, Macchini M, Casadei R, Ricci C, Panzacchi R, Santini D, Biasco G: State of the art biological therapies in pancreatic cancer. World J Gastrointest Oncol 2016;8:5566.

4 Kamisawa T, Wood LD, Itoi T, Takaori K: Pancreatic cancer. Lancet 2016;388:73-85.

5 Bates SE: Pancreatic Cancer: Challenge and Inspiration. Clin Cancer Res 2017;23:1628.

6 Oettle H, Lehmann T: Gemcitabine-resistant pancreatic cancer: a second-line option. Lancet 2016;387:507508.

7 Ward PS, Thompson CB: Metabolic reprogramming: a cancer hallmark even warburg did not anticipate. Cancer Cell 2012;21:297-308.

-8 Sciacovelli M, Frezza C: Metabolic reprogramming and epithelial-to-mesenchymal transition in cancer. FEBS J 2017;284:3132-3144.

-9 Semenza GL: Tumor metabolism: cancer cells give and take lactate. J Clin Invest 2008;118:3835-3837.

10 Birsoy K, Wang T, Possemato R, Yilmaz OH, Koch CE, Chen WW, Hutchins AW, Gultekin Y, Peterson TR, Carette JE, Brummelkamp TR, Clish CB, Sabatini DM: MCT1-mediated transport of a toxic molecule is an effective strategy for targeting glycolytic tumors. Nat Genet 2013;45:104-108.

11 Arora A, Singh S, Bhatt AN, Pandey S, Sandhir R, Dwarakanath BS: Interplay Between Metabolism and Oncogenic Process: Role of microRNAs. Transl Oncogenomics 2015;7:11-27.

12 Wang P, Chen L, Zhang J, Chen H, Fan J, Wang K, Luo J, Chen Z, Meng Z, Liu L: Methylation-mediated silencing of the miR-124 genes facilitates pancreatic cancer progression and metastasis by targeting Rac1. Oncogene 2014;33:514-524.

13 Li KK, Pang JC, Ching AK, Wong CK, Kong X, Wang Y, Zhou L, Chen Z, Ng HK: miR-124 is frequently downregulated in medulloblastoma and is a negative regulator of SLC16A1. Hum Pathol 2009;40:1234-1243.

14 Stoscheck CM: Quantitation of protein. Methods Enzymol 1990;182:50-68.

15 Zhang X, Wu Y, Pan Z, Sun H, Wang J, Yu D, Zhu S, Dai J, Chen Y, Tian N, Heng BC, Coen ND, Xu H, Ouyang $\mathrm{H}$ : The effects of lactate and acid on articular chondrocytes function: Implications for polymeric cartilage scaffold design. Acta Biomater 2016;42:329-340.

16 Liang CC, Park AY, Guan JL: In vitro scratch assay: a convenient and inexpensive method for analysis of cell migration in vitro. Nat Protoc 2007;2:329-333.

17 Wu B, Li S, Sheng L, Zhu J, Gu L, Shen H, La D, Hambly BD, Bao S, Di W: Metformin inhibits the development and metastasis of ovarian cancer. Oncol Rep 2012;28:903-908.

18 Soldani C, Croce AC, Bottone MG, Fraschini A, Biggiogera M, Bottiroli G, Pellicciari C: Apoptosis in tumour cells photosensitized with Rose Bengal acetate is induced by multiple organelle photodamage. Histochem Cell Biol 2007;128:485-495.

19 Stovall DB, Wan M, Zhang Q Dubey P, Sui G: DNA vector-based RNA interference to study gene function in cancer. J Vis Exp 2012;10.3791/4129e4129. 


\section{Cellular Physiology Cell Physiol Biochem 2018;50:924-935 \begin{tabular}{ll|l} 
and Biochemistry Published online: 23 October 2018 & $\begin{array}{l}\text { ○ 2018 The Author(s). Published by S. Karger AG, Basel } \\
\text { www.karger.com/cpb }\end{array}$ \\
\hline
\end{tabular}}

Wu et al.: Mir-124 Suppressed Cancer by MCT1

20 Gass JD: Comparison of uveal melanoma growth rates with mitotic index and mortality. Arch Ophthalmol 1985;103:924-931.

21 Peng Y, Croce CM: The role of MicroRNAs in human cancer. Signal Transduct Target Ther 2016;1:15004.

-22 Silber J, Lim DA, Petritsch C, Persson AI, Maunakea AK, Yu M, Vandenberg SR, Ginzinger DG, James CD, Costello JF, Bergers G, Weiss WA, Alvarez-Buylla A, Hodgson JG: miR-124 and miR-137 inhibit proliferation of glioblastoma multiforme cells and induce differentiation of brain tumor stem cells. BMC Med 2008;6:14.

-23 Ando T, Yoshida T, Enomoto S, Asada K, Tatematsu M, Ichinose M, Sugiyama T, Ushijima T: DNA methylation of microRNA genes in gastric mucosae of gastric cancer patients: its possible involvement in the formation of epigenetic field defect. Int J Cancer 2009;124:2367-2374.

24 Li L, Luo J, Wang B, Wang D, Xie X, Yuan L, Guo J, Xi S, Gao J, Lin X, Kong Y, Xu X, Tang H, Xie X, Liu M: Microrna-124 targets flotillin-1 to regulate proliferation and migration in breast cancer. Mol Cancer 2013;12:163.

25 Zhang H, Wang Q Zhao Q, Di W: MiR-124 inhibits the migration and invasion of ovarian cancer cells by targeting SphK1. J Ovarian Res 2013;6:84.

-26 Zhang J, Lu Y, Yue X, Li H, Luo X, Wang Y, Wang K, Wan J: MiR-124 suppresses growth of human colorectal cancer by inhibiting STAT3. PLoS One 2013;8:e70300.

27 Chen Z, Liu S, Tian L, Wu M, Ai F, Tang W, Zhao L, Ding J, Zhang L, Tang A: miR-124 and miR-506 inhibit colorectal cancer progression by targeting DNMT3B and DNMT1. Oncotarget 2015;6:38139-38150.

28 Zhou L, Xu Z, Ren X, Chen K, Xin S: MicroRNA-124 (MiR-124) Inhibits Cell Proliferation, Metastasis and Invasion in Colorectal Cancer by Downregulating Rho-Associated Protein Kinase 1(ROCK1). Cell Physiol Biochem 2016;38:1785-1795.

-29 Tennant DA, Duran RV, Gottlieb E: Targeting metabolic transformation for cancer therapy. Nat Rev Cancer 2010;10:267-277.

-30 Halestrap AP: The SLC16 gene family - structure, role and regulation in health and disease. Mol Aspects Med 2013;34:337-349.

-31 Eilertsen M, Andersen S, Al-Saad S, Kiselev Y, Donnem T, Stenvold H, Pettersen I, Al-Shibli K, Richardsen E, Busund LT, Bremnes RM: Monocarboxylate transporters 1-4 in NSCLC: MCT1 is an independent prognostic marker for survival. PLoS One 2014;9:e105038.

-32 Pertega-Gomes N, Vizcaino JR, Felisbino S, Warren AY, Shaw G, Kay J, Whitaker H, Lynch AG, Fryer L, Neal DE, Massie CE: Epigenetic and oncogenic regulation of SLC16A7 (MCT2) results in protein over-expression, impacting on signalling and cellular phenotypes in prostate cancer. Oncotarget 2015;6:21675-21684.

33 Lee JY, Lee I, Chang WJ, Ahn SM, Lim SH, Kim HS, Yoo KH, Jung KS, Song HN, Cho JH, Kim SY, Kim KM, Lee S, Kim ST, Park SH, Lee J, Park JO, Park YS, Lim HY, Kang WK: MCT4 as a potential therapeutic target for metastatic gastric cancer with peritoneal carcinomatosis. Oncotarget 2016;7:43492-43503.

-34 Gallagher-Colombo S, Maminishkis A, Tate S, Grunwald GB, Philp NJ: Modulation of MCT3 expression during wound healing of the retinal pigment epithelium. Invest Ophthalmol Vis Sci 2010;51:5343-5350.

-35 Semenza GL: Expression of hypoxia-inducible factor 1: mechanisms and consequences. Biochem Pharmacol 2000;59:47-53.

-36 Arechaga-Ocampo E, Lopez-Camarillo C, Villegas-Sepulveda N, Gonzalez-De la Rosa CH, Perez-Anorve IX, Roldan-Perez R, Flores-Perez A, Pena-Curiel O, Angeles-Zaragoza O, Rangel Corona R, Gonzalez-Barrios JA, Bonilla-Moreno R, Del Moral-Hernandez O, Herrera LA, Garcia-Carranca A: Tumor suppressor miR-29c regulates radioresistance in lung cancer cells. Tumour Biol 2017;39:1010428317695010. 